\title{
Das Betreuungsgesetz droht zu scheitern
}

\author{
Erforderlich sind eine konsequente Professionalisierung, mehr Kompetenzen \\ für die Betreuungsbehörden, obligatorische Sozialgutachten sowie eine \\ regelmäßige Berichterstattung
}

Wolf Crefeld

Rund 1,2 Millionen behinderte oder psychisch kranke Menschen sind in Deutschland auf gesetzliche Betreunng angewiesen. Die zu Recht als Jahrhundertgesetz gepriesene Reform weist jedoch in der Praxis gravierende Vollzugsdefizite auf, die schleunigst zum Handeln zwingen sollten.

Es sind harte Worte, die Heribert Prantl, Jurist und Redakteur der Süddeutschen Zeitung, zur aktuellen Situation des Betreuungsrechts gebraucht:

"Selten wurde ein Gesetz so gelobt, und selten ist ein Gesetz so grandios gescheitert. Es fehlt am politischen Willen zur Umsetzung der neuen Vorschriften - die Spardebatte kommt da gerade recht. Man hat eine aufwendige Reform Gesetz werden lassen, mag aber kein Geld dafür ausgeben. So wird ein Recht zerstört, das anscheinend bereits wenige Jahre nach seiner Verabschiedung nicht in die Zeit passt."

Während viele Psychiatriebetroffene, aber auch mancher Psychiatriereformer meinen, es habe sich durch das Betreuungsrecht in der Praxis nicht allzu viel geändert, vernehmen es andere mit Verwunderung. Schließlich ist doch das Betreuungsgesetz von 1990 immer wieder als "Jahrhundertreform " gefeiert worden. Als ein wesentliches Ergebnis der 1975 durch die Psychiatrie-Enquete in Gang gebrachten Psychiatriereform sollte es psychisch kranke Menschen vom Stigma des Entmündigtseins und der scheinbaren Rechtlosigkeit befreien. An seinem Entwurf wirkte nicht zuletzt Caspar Kulenkampff mit, Vorsitzender der Sachverständigenkommission des Deutschen Bundestages zur Lage der Psychiatrie und

Prof. Dr. Wolf Crefeld lehrte bis zu seiner Pensionierung Sozialmedizin an der Evangelischen Fachhochschule Bochum

E-Mail crefeld@web.de
Begründer der psychiatriepolitisch Einfluss nehmenden »Aktion Psychisch Kranke e. V.«. So entstand 1990 ein zivilund verfahrensrechtliches Gesetzeswerk, das psychisch kranke und geistig behinderte Menschen als Träger der das Menschenbild des Grundgesetzes konkretisierenden Grundrechte wahrnimmt. Es soll Diskriminierung beseitigen sowie Rechte und deren verfahrensrechtliche Durchsetzung stärken. Im Mittelpunkt seiner Zielsetzung steht das Wohl der Betroffenen, ihre persönliche Betreuung und die Stärkung der Personensorge.

Doch Gesetze setzen sich nicht von selbst durch, das gilt ganz besonders, wenn sie zugunsten von Menschen geschaffen werden, die aufgrund der Art ihrer Behinderung nur über geringe oder gar keine politische Artikulationsfähigkeit verfügen. Karl Hermann Haack, bis 2005 Beauftragter der Bundesregierung für die Belange behinderter Menschen, hat das Problem mit der These gekennzeichnet: "Nicht die Formulierung und die Verabschiedung von Gesetzen entscheiden über die Lebenswirklichkeit der Betroffenen, sondern ihre Umsetzung. " Im gleichen Sinne stellte Bernd Schulte auf dem 6. Vormundschaftsgerichtstag fest, dass nicht die positiv-rechtliche Verankerung von Rechten, sondern deren Erfüllung und Durchsetzung hierzulande das Problem sei.

Manche haben schon vor dem Inkrafttreten der Betreuungsrechts vor dessen Scheitern in der Praxis gewarnt. So wiesen renommierte Juristen wie Werner Bienwald und Gisela Zenz darauf hin, dass viele Missstände gar nicht dem geltenden Vormundschafts- und Pflegschaftsrecht anzulasten, sondern eine Folge der Missachtung des damals bereits geltenden Rechts seien.

Angesichts der von ihr in einer Studie für das Bundesjustizministerium belegten Vollzugsdefizite des geltenden Rechts forderte Gisela Zenz neben der zivil- und verfahrensrechtlichen Reform einen gleichrangig zu behandelnden sozialrechtlichen Reform- ansatz, ohne den die Rechtsreform wirkungslos bleiben werde. Im gleichen Sinne mahnte Bernd Schulte, die sozialrechtlichen Voraussetzung dafür zu schaffen, dass die im Betreuungsrecht definierte Hilfeleistung im erforderlichen Umfang und in der erforderlichen Qualität verfügbar ist.

Konkret gehe es um die Gewährleistung der im Einzelfalls jeweils erforderlichen Qualifikation, Beratung, Kontrolle und finanzielle Absicherung der Helfer. Und Rainer Pitschas wies noch vor dem Inkrafttreten des Betreuungsgesetzes kritisch darauf hin, dass die Durchsetzung der Rechtsreform entscheidend vom Aufbau und der Förderung eines Betreuungsnetzwerks aus den wesentlichen Akteuren des Betreuungswesens abhänge. Es seien vor allem die näheren organisatorischen Bedingungen und das je feldspezifische institutionelle Ambiente, die den Prozess der Rechtsverwirklichung und damit jede Rechtsreform beeinflussen.

Nachdem das Betreuungsgesetz 1992 in Kraft getreten war, entstanden mancherorts fast chaotische Zustände. Denn im Wesentlichen trug zwar die Justiz die Verantwortung für die Umsetzung der Reform, doch war sie auf diese für sie ungewohnte Aufgabe nicht ausreichend vorbereitet. Die gemäß den Zielen des Betreuungsrechts an sie gerichtete Forderung nach einem anderen Selbstverständnis, einem »neuen Richterbild « (von Looz), passt kaum zur tradierten Juristenausbildung und den Arbeitsstrukturen der Justiz, die vor allem auf Konfliktschlichtung ausgerichtet sind.

Die Folge ist eine regional erheblich divergierende Anwendungspraxis, die sich sowohl in der Einstellung gegenüber der Klientel wie auch in der Bereitschaft zur Kooperation mit den anderen Akteuren des Betreuungswesens ausdrückt. Von den seit 1992 entstandenen kommunalen Betreuungsbehörden mögen manche gut, andere sicher kläglich für ihr wenig klares Aufgabenprofil gerüstet sein. Dementsprechend unterschiedlich ist ihre Aufgabenwahrnehmung. 
Seit dem Inkrafttreten des Betreuungsrechts entwickelte sich zunächst ein Wildwuchs meist freiberuflich tätiger Betreuer, manche hoch engagiert und fachlich qualifiziert - ein Drittel mit einem Studium der Sozialarbeit -, während andere mit den unterschiedlichsten oder gar keinen Ausbildungen und Berufserfahrungen einfach einen Job suchten, ohne auch nur den Sinn des neuen Rechtsinstituts verstanden zu haben. Die Zahl der Betreuungen verdoppelte sich in zehn Jahren, zugleich trieben aber auch zahllose Vergütungsstreitigkeiten die Kosten für die Justizhaushalte in die Höhe. Die Folge waren zwei Betreuungsrechtsänderungsgesetze, die vorrangig das Ziel der Kostensenkung verfolgten, ohne an den strukturellen Mängeln wesentliches zu ändern.

Inzwischen ist es vor allem die Praxis, die sich über ihre Verbände und Arbeitsgemeinschaften darum sorgt, dass das Betreuungswesen den gesetzten Zielen doch noch gerecht wird. So haben die Verbände der berufsmäßig tätigen Betreuer gemeinsam ein Berufsbild formuliert mit dem Ziel der Professionalisierung des Berufs. Deshalb engagieren sie sich für die berufsfachliche Qualifizierung ihrer Mitglieder mittels Fortbildung und weiterbildenden Studiengängen, einer Berufsordnung, der Entwicklung von Handlungsleitlinien und der Schaffung eines Kompetenz nachweisenden Berufsregisters. Ob die Gerichte diese Qualitätsbemühungen zur Kenntnis nehmen, ist nach den bisherigen Erfahrungen keineswegs sicher.

Aus der Mitte der Betreuungsbehörden sind ein "Anforderungsprofil für örtliche Betreuungsbehörden « und »Orientierungshilfen zur Umsetzung des Betreuungsrechts « formuliert worden (siehe Kania u.a. 2006). Wieweit allerdings diese Empfehlungen in den einzelnen Behörden Realität werden, hängt wesentlich von der Kooperationsbereitschaft der örtlichen Gerichte und der Neigung der Kommunalverwaltungen ab, ihrer Behörde eine aufgabenangemessene Ausstattung zu geben. Denn solange die Betreuungsbehörden vom Gesetzgeber keine klare Aufgabenzuweisung und entsprechende Durchsetzungsmöglichkeiten erhalten, sind sie auf die (nicht immer vorhandene) Kooperationsbereitschaft der anderen Akteure angewiesen.

Angesichts dieser insgesamt unerfreulichen Situation wurde 1998 auf Initiative der Abgeordneten und früheren Familienrichterin Margot von Renesse von der SPD-Fraktion des Deutschen Bundestages ein Antrag zur Reform des Betreuungsrechts eingebracht (Bundestags-Drucksache 13/10301). Darin heißt es, das geltende Betreuungsrecht werde seinen Reformzielen nicht in ausreichendem Maße gerecht. Entscheidende Ursache dafür sei, dass der Gesetzgeber seine Vorstellungen weitgehend mit den Mitteln des Zivilrechts und des justiziellen Instrumentariums habe durchsetzen wollen, obgleich beide nur in Grenzen dafür tauglich seien. Deshalb forderten die Abgeordneten ein neu zu schaffendes "Betreuungshilfegesetz « als Teil des Sozialgesetzbuches, in dem die Infrastruktur zur Umsetzung des Betreuungsgesetzes verbindlich geregelt werden sollte. Insbesondere soll eine Aufgabenverlagerung von der Justiz zur Betreuungsbehörde statt finden, die dann Steuerungsfunktionen mit dem Ziel eines wirksam arbeitenden örtlichen Betreuungswesens übernehmen soll.

Der Antrag führte zwar in der folgenden Sitzungsperiode zu einer interfraktionellen Arbeitsgruppe, wurde dann aber überrollt von einer Bundesratsiniti-

\section{Monitoring}

In der Rubrik » Monitoring « nehmen Autorinnen und Autoren einzelne Arbeits- und Themenfelder der Sozialen Arbeit in den Blick um zu untersuchen, was sich dort Neues tut oder warum dort Praxis und Theorie auf der Stelle treten. Die Artikel sind eine aktuelle Ergänzung zu den wechselnden Schwerpunktthemen aus der Sozialen Arbeit in den Blättern der Wohlfahrtspflege. Eine Übersicht der bisherigen Beiträge in der Rubrik "Monitoring «:

Was Fachkräfte wissen müssen

Der Qualifikationsrahmen Soziale Arbeit

Von Peter Buttner und Ulrich Bartosch

Blätter der Woblfahrtspflege 1/2007, Seite 28-30

Das Format mit Inhalt füllen

Interview mit Wolf Rainer Wendt zum »Qualifikationsrah-

men Soziale Arbeit «

Blätter der Woblfabrtspflege 1/2007, Seite 29

Eltern stützen, Kinder schützen

Nach den Gesetzesänderungen:

Wie zukunftsfest ist das Kinder- und Jugendhilferecht?

Von Johannes Münder

Blätter der Wohlfahrtspflege 1/2007, Seite 31-32

Alles wird zur Dienstleistung

Die wirkungsorientierte Finanzierung von Jugendhilfe

Von Benjamin Landes

Blätter der Wohlfahrtspflege 1/2007, Seite 33-34
Schulsozialarbeit braucht mehr Rechte

Ein Berufsfeld der Sozialen Arbeit zwischen Lerntradition und Lebenswelt

Von Susanne Hartmann-Hanff

Blätter der Woblfahrtspflege 1/2007, Seite 35-36

Qualitätssicherung zivilisiert den Wettbewerb

System und Arbeitsfelder im Vergleich

Von Roland Schmidt

Blätter der Woblfahrtspflege 6/2006, Seite 230-232

Indikatoren gesucht

Neue Ansätze zur Integrationssteuerung in Deutschland

Von Kristin Schwarze

Blätter der Wohlfahrtspflege 6/2006, Seite 233-234

Die armen Kinder

Junge Menschen als die unsichtbaren Verlierer von Hartz IV

Von Gerda Holz

Blätter der Wohlfahrtspflege 5/2006, Seite 193-195

Der Jahresbezugspreis beträgt 58,- Euro (für Studierende und arbeitslose Bezieher auf jährlichen Nachweis 29,-- Euro).

Die Einzelhefte können zum Preis von 11,- Euro zuzüglich Versandkosten und Mehrwertsteuer bezogen werden bei:

Nomos Verlagsgesellschaft, 76520 Baden-Baden, Telefon 07221 2104-39, Fax 07221210443,

E-Mail hohmann@nomos.de 
ative der Länderjustizminister, die einen erneute Anlauf nahmen, die Kosten des Betreuungswesens für die Justiz zu senken. Die notwendigen Diskussionen um eine Strukturreform wollten sie nicht abwarten, sodass der Sozialministerkonferenz der Länder nur noch der Hinweis auf die weiterhin bestehende Notwendigkeit einer Strukturreform blieb. In ihrem Beschluss vom November 2003 stellten anderen Wissenschaftlern insbesondere Rainer Pitschas und Rolf Marschner die Diskussion um eine im Sozialrecht verankerte Strukturreform des Betreuungswesens voran getrieben. Auch Pitschas, Professor an der Deutschen Hochschule für Verwaltung in Speyer, fordert ein $\gg$ Betreuungshilferecht «.

Betreuung dürfe nicht nur als Antragsund Rechtsverhältnis begriffen werden, in

\section{»Es fehlt nicht an Gesetzen, sondern an ibrer Umsetzung "}

sie insbesondere Regelungsbedarf fest für die Entwicklung eines originären $\mathrm{Zu}$ ständigkeitsprofils der Betreuungsbehörden, denen unter anderem eine Aufsichtsfunktion zu übertragen sei, sowie für eine fortlaufenden Berichterstattung zur Lage des Betreuungswesens, wie sie sich in vielen Politikbereichen inzwischen bewährt hat.

Seither haben der Vorstand des Vormundschaftsgerichtstags in seinen 1999 veröffentlichten Leitlinien sowie neben

\section{Literatur}

Kania, M. et al. (2006). Die Rolle der Betreuungsbehörden und die Perspektiven einer weiteren Entwicklung. Soziale Arbeit 7-8.2006: 254-260.

Looz, C. v. (2006). Das neue Richterbild in Betreuungssachen. Soziale Arbeit 7-8/2006: 283-289.

Marschner, R. (2004). Betreuungsrecht und Sozialgesetzbuch. Möglichkeiten der Koordination und Kooperation. Betrifft: Betreuung Bd. 7: 122-131.

Pitschas, R. (2004). Gesetzliche Betreuung im Wandel. Eindrücke, Wahrnehmungen und Ausblicke nach 11 Jahren Betreuungsrecht. In: Brill, K. E. (Hg.). Betreuungsrecht in Bedrängnis. Betrifft: Betreuung Bd. 7: 132-143.

Schulte, B. (1999) Grundrechtsgarantien und ihre Einlösung. Ein Blick zurück und nach vorn auf die Entwicklung des Betreuungsrechts im Spannungsfeld von Rechtsfürsorge und Sozialstaat. Betrifft: Betreuung Bd. 1: 8-18.

Vormundschaftsgerichtstag e. V. (1999).

Leitlinien zur rechts- und sozialpolitischen Diskussion um die Weiterentwik-

klung des Betreuungsrechts. Betreuungsrechtl. Praxis 4/1999:123-125. dem eine Person für eine andere Geschäfte zu besorgen hat. Stattdessen sei immer wieder daran zu erinnern, dass das Betreuungsrecht seiner ureigenen Funktion nach Sozialrecht darstelle. Dazu sei den kommunalen Betreuungsbehörden ein entsprechender Qualitätssicherungsauftrag zu erteilen. Verschiedene Autoren verweisen auf die Parallele zum Kindschafts- und Jugendrecht hin, in dem das Miteinander von staatlicher Hilfe und staatlichem Eingriff einerseits im Kinderund Jugendhilferecht (SGB VIII), andererseits im Bürgerlichen Gesetzbuch geregelt ist.

Für das Betreuungswesen seien Regelungen notwendig zu einer sachgerechten Verknüpfung des Betreuungsrechts mit dem Sozialleistungsrecht, insbesondere dem SGB IX. Marschner, Mitherausgeber der Zeitschrift Recht \& Psychiatrie, fordert dazu einen Zuwachs an fachlicher und rechtlicher Kompetenz in den Betreuungsbehörden, damit im Einzelfall wirksamer geprüft werden kann, ob durch die Initiierung von Hilfen aus dem Sozialgesetzbuch auf die Bestellung eines Betreuers verzichtet werden kann.

\section{Was getan werden muss}

Nach dem Stand der Diskussion lassen sich vor allem die folgenden Aufgaben für den Gesetzgeber benennen:

1 Die Qualität der Betreuungsarbeit ist - sicher zu stellen. Der Betreuerberuf bedarf der konsequenten Professionalisierung, sodass die Entwicklung der Kriterien für die fachliche Eignung zu einer Aufgabe der berufsständischen Selbstver- waltung werden kann. Ehrenamtliche Betreuer bedürfen entsprechender Unterstützung durch Betreuungsvereine und Behörden. Im Übrigen sollte es Aufgabe der Betreuungsbehörde als sozialpädagogischer Fachbehörde werden, auf die Eignung der am Ort tätigen Betreuer in $\mathrm{Zu}$ sammenarbeit mit dem Gericht verbindlich hinzuwirken.

2. Für die Betreuungsbehörden sind - Steuerungsaufgaben im örtlichen Betreuungswesen verbindlich zu definieren, sodass sie für die Qualität der dort geleisteten Arbeit wirksam sorgen können.

3 Um den Nachrang der Betreuung - gegenüber anderen sozialen Hilfen sicher zu stellen, sind die Betreuungsbehörden obligat vor jeder Betreuerbestellung zu beteiligen. Sie haben zum Hilfebedarf der betroffenen Person Sozialgutachten zu erstellen und sollten dabei festgestellte Rechtsansprüche gegenüber Sozialleistungsträgern zwecks Vermeidung nicht erforderlicher Betreuungen Geltung verschaffen. Wenn aber eine Betreuung erforderlich erscheint, sollten sie auf der Basis ihres Assessments die Grundlagen für ein planvolles Betreuungsmanagement durch den vom Gericht zu bestellenden Betreuer legen.

4 Das Betreuungswesen, durch das •1,2 Millionen behinderte oder psychisch kranke Menschen betreut werden, bedarf angesichts der offenkundigen Gefahr, dass wesentliche Vollzugsdefizite den Normalzustand charakterisieren, einer regelmäßigen Berichterstattung für das Ziel einer lernenden Gesetzgebung.

Wenn der Gesetzgeber das "grandiose Scheitern " einer "Jahrhundertreform" noch abwenden will, so sind jetzt neben den Rechtspolitikern insbesondere die Sozialpolitiker und Sozialverwaltungen zum Handeln aufgefordert. 